\title{
Erratum to: Folate-conjugated chitosan-polylactide nanoparticles for enhanced intracellular uptake of anticancer drug
}

\author{
Shengtang Huang • Ying Wan • \\ Zheng Wang $\cdot$ Jiliang Wu
}

Published online: 17 January 2014

(C) Springer Science+Business Media Dordrecht 2014

\section{Erratum to: J Nanopart Res (2013) 15:2096 DOI 10.1007/s11051-013-2096-1}

The original paper "Folate-conjugated chitosan-polylactide nanoparticles for enhanced intracellular uptake of anticancer drug" was published in the Journal of Nanoparticle Research, 2013, 15:2096. The description for the synthesis of folate-conjugated chitosanpolylactide (FA-CH-PCL), which appeared in the page 3 of 15, right column, lines: 11-35, was not correct and is replaced with the following statement.

"Polylactide (PLA) was grafted onto $\mathrm{CH}$ following group-protection methods with some modifications

The online version of the original article can be found under doi:10.1007/s11051-013-2096-1.

S. Huang · J. Wu ( $₫)$

The Cardiovascular, Cerebrovascular and Metabolic Disorder Research Institute, Hubei University of Science and Technology, Xianning 437100, People's Republic of China

e-mail: xywj1@163.com

Y. Wan $(\bowtie)$

College of Life Science and Technology, Huazhong

University of Science and Technology, Wuhan 430074,

People's Republic of China

e-mail: ying_x_wan@yahoo.ca

\section{Z. Wang}

School of Pharmaceutical Science and Technology,

Tianjin University, Tianjin 300072, People's Republic of China
(Duan et al. 2010; Zhou et al. 2013; Feng and Dong 2007). In a typical procedure, $1 \mathrm{~g}$ of $\mathrm{CH}$ was dissolved in $30 \mathrm{~mL}$ of methanesulfonic acid, followed by addition of $2.57 \mathrm{~g}$ of LA monomer and $0.73 \mathrm{~g}$ of 4-dimethylaminopyridine. The reaction was allowed to perform for $12 \mathrm{~h}$ at $120{ }^{\circ} \mathrm{C}$ with stirring while bubbling with nitrogen. The resulting mixture was then transferred to a beaker that contained $200 \mathrm{~mL}$ of $0.2 \mathrm{M} \mathrm{KH}_{2} \mathrm{PO}_{4}, 20 \mathrm{~mL}$ of $4 \mathrm{M} \mathrm{NaOH}$ and was cooled in a bath $\left(0{ }^{\circ} \mathrm{C}\right)$ to remove the acid residue. The collected precipitate was extracted with toluene to remove PLA homopolymers. Afterward, the product was dialyzed against distilled water for 2 days using a membrane tube (MW cutoff: $10 \mathrm{kD}$ ) and lyophilized for further use. By mainly changing the feed ratio of LA to $\mathrm{CH}$, several types of CH-PLA copolymers were synthesized using the same protocol. Folate was then conjugated onto CH-PLAs using the method as the same as that described in the synthesis of FA-CH and FA-substitution degree for FA-CH-PLAs was controlled around $10 \mathrm{wt} \%$ or lower." 